\title{
Asymmetric life-history decision-making in butterfly larvae
}

\author{
Magne Friberg • Inger M. Aalberg Haugen • \\ Josefin Dahlerus · Karl Gotthard · Christer Wiklund
}

Received: 5 May 2010/Accepted: 23 September 2010/Published online: 17 October 2010

(c) The Author(s) 2010. This article is published with open access at Springerlink.com

\begin{abstract}
In temperate environments, insects appearing in several generations in the growth season typically have to decide during the larval period whether to develop into adulthood, or to postpone adult emergence until next season by entering a species-specific diapause stage. This decision is typically guided by environmental cues experienced during development. An early decision makes it possible to adjust growth rate, which would allow the growing larva to respond to time stress involved in direct development, whereas a last-minute decision would instead allow the larva to use up-to-date information about which developmental pathway is the most favourable under the current circumstances. We study the timing of the larval pathway decision-making between entering pupal winter diapause and direct development in three distantly related butterflies (Pieris napi, Araschnia levana and Pararge aegeria). We pinpoint the timing of the larval diapause decision by transferring larvae from first to last instars from long daylength (inducing direct development) to short daylength conditions (inducing diapause), and vice versa. Results show that the pathway decision is typically made in the late instars in all three species, and that the ability to switch developmental pathway late in juvenile life is conditional; larvae more freely switched from diapause to direct development than in the opposite direction. We contend that this asymmetry is influenced by the additional physiological preparations needed to survive the long and cold winter period, and that the reluctance to make a late
\end{abstract}

Communicated by Klaus Fischer.

M. Friberg $(\bowtie)$ I. M. Aalberg Haugen · J. Dahlerus

K. Gotthard · C. Wiklund

Department of Zoology, Stockholm University,

10691 Stockholm, Sweden

e-mail: magne.friberg@zoologi.su.se decision to enter diapause has the potential to be a general trait among temperate insects.

Keywords Diapause/direct development - Seasonal polyphenism · Phenotypic plasticity · Lepidoptera . Developmental constraints

\section{Introduction}

Decision-making is ubiquitous in nature, and has typically been studied over short time scales, for example during foraging (Stephens and Krebs 1986), mating events (Andersson 1994), or in the choice of egg substrates (Jaenike 1978). The evolution of decision-making is most often studied in the context of optimality models, and the genetic architecture of individuals behaving "optimally" is predicted to be favoured by selection (Krebs and Davies 1993; McNamara and Houston 1996). Indeed, there are a great many works in the literature that provide evidence for optimality in nature (Krebs and Davies 1993; Alcock 2009), implying a strong selection on decision-making mechanisms (Stearns 1992; Stearns and Hoekstra 2000; Gotthard 2008). However, some decisions are made well before they become functional. Some of these decisions are "crossroad decisions" that are made early in life and affect growth and development patterns as well as the life history of the individual (Gotthard 2008). One example of such a decision with long-term implications for an individual's life history is the larval pathway decision of temperate insects: whether to continue growth and development into the adult stage during the same season or postpone adult emergence until the next season by arresting growth and development and entering diapause before the onset of harsh winter conditions (Gotthard 2008). 
Temperate insects can have one or several reproductive generations per season, but typically spend the inhospitable winter period in a species-specific diapause resting state (Danilevskii 1965). Species overwintering as eggs are typically either obligatory univoltine (e.g. Carriere et al. 1996; Saulich and Musolin 1996) or have a maternally influenced diapause determination (e.g. Koevos and Tzanakakis 1991; Shintani and Higuchi 2008). In bi- or multivoltine species overwintering as large larvae, pupae or adults, each individual must decide whether to develop directly or delay reproduction until next season. This decision is typically dependent on the conditions experienced by the larvae (Danilevskii 1965). In general, individuals develop directly into reproductively active adults if their offspring are likely to have time to reach the speciesspecific diapause stage before growth and development is hampered by the onset of winter. Direct development is favoured early in the reproductive season and diapause development later in the season, and during a time window in-between, some individuals enter one developmental pathway and some the other (Wiklund et al. 1992).

The larval pathway decision is typically dependent on seasonal cues such as daylength (e.g. Danilevskii 1965; Solbreck 1979; Yata et al. 1984; Eizaguirre et al. 1994; Friberg et al. 2008), temperature (e.g. Danilevskii 1965; Nylin 1992; Eizaguirre et al. 1994; Wedell et al. 1997; Friberg and Wiklund 2010), and in some phytophagous species also on the larval host plant (Wedell et al. 1997; Hunter and McNeil 1997; Friberg et al. 2008; Friberg and Wiklund 2009, 2010). Long daylength and high temperatures provide cues for early season and direct development, whereas shorter daylengths and lower temperatures provide cues that winter is approaching and promote diapause development (Wiklund et al. 1991, 1992).

The larval decision whether to enter diapause or direct development has been described as a "developmental switch" (sensu West-Eberhard 2003) that initiates downstream induction of generation-specific differences in life history, morphology and behaviour (Gotthard 2008). Whereas much research attention has been devoted to the adaptive significance of having different seasonal morphs (e.g. Shapiro 1976; Tauber et al. 1986; Moran 1992; Kingsolver 1995), the evolutionary and ecological significance of the decision mechanism has not been given the same attention. Likewise, the proximate causes of the diapause induction in terms of the cues of importance (see above) and its proximate determination (e.g. Danilevskii 1965; Hodkova and Hodek 2004) are well understood in a few model systems, but for most species little is known about when (i.e. the developmental stage or larval instar) the pathway decision is made (Danks 2007). The available evidence consists of scattered data from different insect groups (reviewed in Danks 1987), and for butterflies, which are the prime targets of this study, the diapause decision-making is best studied in the closely related pierids Pieris rapae (Barker et al. 1963) and Pieris brassicae (Danilevskii 1965). In these species, which overwinter in the pupal stage, the evidence suggests that the decision is made in the ultimate or penultimate larval instars. Some other insects make their pathway decision as young larvae, and in yet others the decision point is located just before entering diapause (Danks 1987, 2007).

Furthermore, previous studies dealing with the timing of the pathway decision in insects are typically descriptive, and rarely include an evolutionary perspective on the decision-making. It is possible to envisage both costs and benefits of deciding early on, and of waiting until the last minute, since an early decision makes it possible to adjust growth rate in good time to reach the reproductive stage while it is still favourable (Abrams et al. 1996), whereas a last-minute decision allows the larvae to use up-to-date information about how they are positioned in the season and whether it is favourable to develop directly or enter diapause (Danks 2007).

It is also largely unknown whether the same processes act in parallel in different species experiencing similar life histories in terms of diapause strategy. In this study, we investigate the diapause decision in three distantly related butterflies that all have the ability to overwinter in the pupal stage, and that have all been used as model organisms in studies of seasonal polyphenism in morphology, behaviour and colouration. We study one pierid butterfly, the greenveined white (Pieris napi), and two nymphalid butterflies from different subfamilies: the satyrine speckled wood butterfly (Pararge aegeria) and the map butterfly (Araschnia levana) belonging to the subfamily Nymphalinae. Without forestalling a future phylogenetic analysis of the evolution of diapause stages in butterflies, the ability to undergo pupal diapause in these three species is likely to have originated separately; the closest temperate relatives to A. levana overwinter either as adults (e.g. Inachis io and Aglais urticae: Wiklund et al. 2007) or are migratory and lack a proper diapause (e.g. Vanessa atalanta: Stefanescu 2001; Mikkola 2004; and Vanessa cardui: Stefanescu 2007; Nesbit et al. 2009). Apart from a closely related species (Lasiommata petropolitana: Gotthard 1998), P. aegeria is the only species among the satyrines in the Northern hemisphere with the ability to enter pupal diapause, and although most temperate relatives to $P$. napi enter pupal diapause, other pierids-including the fellow pierine Aporia crataegi-diapause as young larvae (Eliasson et al. 2005). Hence, the distant relationship between the three species of interest makes it possible to tentatively assess the generality of the processes shaping diapause decisionmaking mechanisms in growing butterfly larvae.

In this study, we pinpoint the timing of the larval diapause decision in our three study species. By transferring 
larvae between light regimes and recording their propensity to enter diapause or direct development, we show several similarities in life-history decision-making among the species, and highlight how adaptive and nonadaptive processes most likely interact to shape the larval diapause decision in temperate butterflies.

\section{Materials and methods}

\section{Study species}

The green-veined white (P. napi) is a widespread butterfly with subspecies covering most of the Northern Hemisphere. In Sweden, this butterfly has three generations in the south, 2-3 generations in central Sweden and is univoltine in the northernmost part of the distribution (Eliasson et al. 2005). Eggs are placed singly on a suite of crucifers, and the larvae go through five instars before pupation.

The map butterfly (A. levana) is spread almost throughout Eurasia, and is currently expanding its distribution in Europe in both the north and south directions. In Sweden, this species arrived at Skåne (the southernmost county) 10-15 years ago, and the map butterfly is now established throughout the southern third of the country, where they appear in two generations per year. This species is also well known for being seasonally polyphenic, with the spring generation having an orange dorsal wing pattern and the summer generation being strikingly differentblackish with a white band traversing the dorsal side of the wings (e.g. Windig and Lammar 1999). Eggs are laid in clutches of 8-15 on stinging nettle (Urtica dioica), and larvae develop through five instars before pupation.

In Sweden, the speckled wood butterfly ( $P$. aegeria) has a disjunct distribution, with a southern population having immigrated into Sweden from the south in 1939 and a northern population that has been present for as long as there are records (Nordström 1955). Interestingly, the two populations differ in life histories, as they appear in 4-5 cohorts per year in the south, with a complicated lifecycle (Van Dyck and Wiklund 2002; Wiklund and Friberg 2010), as they are able to overwinter as either pupae or third-instar larvae (Wiklund and Friberg 2010). This leads to a complex phenology with individuals that have spent the winter as pupae eclosing in April and May in a spring flight period. Meanwhile, the overwintering larvae break their diapause and develop into pupae, and about a month later this cohort ecloses into a second flight period. The offspring of two spring cohorts develop directly (without diapause) into two separated summer cohorts (Van Dyck and Wiklund 2002). In central Sweden this species is an obligatory pupal diapauser (Gotthard and Berger 2010), and typically appears in a single large generation per year, but occasionally flies in a partial, small, directly developing summer generation (Eliasson et al. 2005).

\section{Experimental protocol}

\section{Green-veined white (Pieris napi)}

All experimental larvae descended (first- or second-generation offspring) from 16 P. napi females that were collected in the Stockholm area in late July 2008. Females were allowed to lay eggs on their crucifer host plant, garlic mustard (Alliaria petiolata). Eggs were kept at room temperature until they hatched, when the larvae were transferred to the experimental conditions. The experiment was conducted in two replicates. First we performed a large-scale study to detect the broad timing of the larval pathway decision by transferring eggs and larvae of all instars between a long day and a short day treatment, and then we replicated the experiment by only transferring larvae of the last three instars (III-V) (see "Results").

In the first replicate, a total of 254 newly deposited eggs were divided equally between two climate cabinets (Termaks Series KB8000L; Termaks, Bergen, Norway): in one, a long day treatment induced direct development (20:4 h light/dark); in the other, a short day treatment induced diapause (16:8 h light/dark). A constant temperature of $23^{\circ} \mathrm{C}$ was maintained. As the larvae hatched they were kept in pairs in $0.5 \mathrm{~L}$ plastic jars, and they had ad libitum access to their food plant $A$. petiolata. Larvae were inspected on a daily basis, and a fraction of the larvae at each instar were transferred in one of two directions (from short to long day and vice versa). Due to rapid larval growth and some problems with the timing of the transfer of larvae in intermediate instars, sample sizes were uneven, with larvae in instars II-IV moved from a long to a short daylength being somewhat underrepresented. The butterflies were individualized as pupae, and individuals that did not enter direct development were kept at $23^{\circ} \mathrm{C}$ for at least 3 weeks after pupation before they were classified as having entered diapause. A total of 216 of the 254 individuals $(85 \%)$ that were used in the experiment survived into the pupal stage. Direct developers were sexed as adults, whereas diapausers were sexed as pupae after not having eclosed 3 weeks after pupation, taking advantage of the fact that females have two genital slits on the underside of the pupal abdomen, which are easily distinguishable from the single genital slit visible in male pupae (e.g. Jackson 1890).

As the first replicate showed that the larval decision whether to enter direct or diapause development is induced in the later larval instars (III-V; see "Results"), we then focused on these three stages and initially involved 120 larvae from the laboratory stock population (see above). The larvae were divided between the same two climate 
cabinets (short and long daylength treatments), and were reared singly in $0.5 \mathrm{~L}$ jars with constant access to A. petiolata. Larvae were then reciprocally transferred between the two cabinets at the beginning of instar III, IV or V, and all larvae were weighed at transfer to definitely determine their instar affiliation. As above, pupae were kept at $23^{\circ} \mathrm{C}$ for at least three weeks after the pupation event before they were classified as diapause developers. Direct developers were sexed at eclosion, whereas diapausing individuals were sexed as pupae. Survival was high among the larvae, and 111 of the initial 120 larvae $(92.5 \%)$ survived until pupation. In P. napi, as well as in A. levana and P. aegeria, mortality was highest in the first instar, whereas it was possible to score all larvae that reached the pupal stage as having either eclosed into adulthood or decided to diapause 3 weeks after the pupation date.

\section{Map butterfly (Araschnia levana)}

On 29 July 2009, 16 females were collected in Ålstorp (latitude $\mathrm{N} 55^{\circ} 49^{\prime} 0^{\prime \prime}$, longitude E12 $58^{\prime} 0^{\prime \prime}$ ) in southernmost Sweden. Females laid eggs in $1 \mathrm{~L}$ jars with access to the host plant, stinging nettle (Urtica dioica). Egg strains were incubated in Petri dishes on moist filter paper in climate cabinets, maintaining $23^{\circ} \mathrm{C}$ until hatching. Because larvae of this species are gregarious until the penultimate or ultimate instar (Eliasson et al. 2005), the newly hatched larvae were transferred in groups of five into $0.5 \mathrm{~L}$ jars with ad libitum access to stinging nettle into the experimental treatments. In total, 300 newly hatched larvae were divided between a short day $\left(12: 12 \mathrm{~h}\right.$ light/dark, $\left.23^{\circ} \mathrm{C}\right)$ diapause-inducing treatment and a long day (20:4 h light/ dark, $23^{\circ} \mathrm{C}$ ) treatment inducing direct development. In each of the five instars, 30 larvae were transferred in both directions. All larvae were individualized in the fourth instar and reared in single $0.5 \mathrm{~L}$ jars until pupation. Adults were sexed upon eclosion, whereas pupae that were uneclosed 3 weeks after pupation were considered to have entered diapause and were sexed as pupae.

\section{Speckled wood (Pararge aegeria)}

The experiment included larvae from both populations of the disjunct Swedish distribution, and was conducted in two replicates. Before the first replicate, two already mated females from the central Swedish population were collected in the Stockholm area in summer 2008. Offspring of these females were allowed to overwinter as pupae in outdoor conditions on the roof of the biology laboratory buildings at Stockholm University, and taken into the laboratory for eclosion and mating in early April 2009. The first replicate then included 22 larvae from 7 successfully mated central Swedish females tested together with 39 first-generation offspring of 8 females collected at Ransvik, Skåne, in April 2009, representing the southern population. Females were allowed to deposit eggs on annual meadow grass (Роа аппиа) in $1 \mathrm{~L}$ plastic containers, and eggs were incubated at room temperature until hatching. Newly hatched larvae were placed in individual plastic jars $(0.5 \mathrm{~L})$ with ad libitum access to $P$. апnиа in either diapauseinducing short day conditions $\left(15: 9 \mathrm{~h} \mathrm{light} / \mathrm{dark}, 17^{\circ} \mathrm{C}\right)$ or direct development-inducing long day conditions $(21: 3 \mathrm{~h}$ light $/$ dark; $\left.17^{\circ} \mathrm{C}\right)$. Cohorts of larvae were then transferred between experimental conditions in instars II-IV. Directly developing individuals were sexed after eclosion, and diapausing individuals were sexed after having spent 25 days in the pupal stage without eclosing into adults. The second replicate followed the same protocol and included 64 offspring larvae from 13 females collected in the Stockholm area in June 2009. Based on results from the first replicate, larvae were transferred between experimental conditions in the penultimate (III) or ultimate (IV) instar (see "Results").

\section{Statistical analysis}

The timing of the larval pathway decision was initially explored for each species in logistic regression models using pathway (diapause $=0$, direct development $=1$ ) as a binomially distributed response variable with logit as link function. We divided each dataset between larvae transferred from long to short daylength and those transferred in the opposite direction, as the transfer directions were designed to differently affect the larval pathway decision. In each model we included sex and instar as categorical predictor variables when a larva was moved between daylengths. For $P$. aegeria we also included population as an initial categorical factor.

In order to allow species to be compared, and to detect the impacts of both the instar of transfer and the transfer direction, we tested for differences in larval ability to switch from one pathway to the other by comparing how late in life the larvae could be transferred from a short day to a long day, or vice versa, and still adjust their pathway choice (diapause/direct development) to fit the new environment. We did this in separate models for each species by scoring each individual as having made the decision based on information received in the old cabinet (0) or in the new cabinet (1), which created a binomially distributed response variable. This means that a larva that was transferred to the long day treatment and which later entered diapause had already made its pathway decision before the transfer and was thus assigned a zero (0), whereas a larva that was transferred in the same direction and entered direct development was assigned the value of one (1). Analogously, larvae that were transferred from the long day to the short day treatment but entered direct development 
were scored as having made their decision before the transfer (0), whereas larvae that entered diapause development were assigned as having made the decision under the new light period conditions (1). The effects of the categorical predictors sex, instar of transfer (IV or $\mathrm{V}$ for $P$. napi and A. levana and instar III and IV for P. aegeria) and transfer direction (long day $\rightarrow$ short day/short day $\rightarrow$ long day) were tested by applying logistic regressions with logit as link function. For $P$. napi and P. aegeria we also included the effect of replicate in the initial model, and for $P$. aegeria we also tested whether the population that the experimental larvae descended from (northern or southern population) had an effect. Nonsignificant factors and interactions were sequentially dropped from the models.

\section{Results}

The larval responses to transfer between daylengths were highly similar between species (Table 1). In all species, virtually all larvae that were transferred to the short daylength in the early instars decided to enter diapause, whereas larvae transferred from short to long daylengths as young larvae entered direct development. Larvae transferred later during development were either able to adjust their decisions to fit the new circumstances, or they had already made their decisions in the original light environment (Table 1). The sex of the individual did not affect the timing of the pathway decision in any of the three species when transferred into the long day treatment (Table 1), and
Table 1 Sample sizes and pathway destinies for larvae of P. napi, A. levana and $P$. aegeria that were transferred between long day and short day treatments in different instars (left side of table); the models obtained from a logistic regression with pathway [diapause (0), direct (1)] used as response variable and the sex of each larva and the instar of transfer used as predictor variables (right side of table)
Significant differences are highlighted in bold

a Six of 13 males entered diapause whereas only 1 of 18 females entered diapause when transferred to the short day treatment in the ultimate instar

${ }^{b}$ No variation in dataset

\begin{tabular}{|c|c|c|c|c|c|c|c|}
\hline \multirow[t]{2}{*}{ Instar } & \multicolumn{2}{|c|}{ Pathway } & \multirow[b]{2}{*}{ Dir. dev. (\%) } & \multicolumn{4}{|c|}{ Statistics (log. regr.) } \\
\hline & Direct & Diapause & & & $\chi^{2}$ & $d f$ & $P$ \\
\hline \multicolumn{8}{|l|}{ P. napi } \\
\hline \multicolumn{8}{|c|}{ Long day $\rightarrow$ short day } \\
\hline I & 1 & 29 & 3.3 & & & & \\
\hline II-III & 2 & 32 & 6.3 & Sex & 0.29 & 1 & 0.5886 \\
\hline IV & 11 & 24 & 31.4 & Instar moved & 86 & 4 & $<0.001$ \\
\hline $\mathrm{V}$ & 49 & 7 & 87.5 & & & & \\
\hline \multicolumn{8}{|c|}{ Short day $\rightarrow$ long day } \\
\hline I & 26 & 0 & 100 & & & & \\
\hline II-III & 44 & 1 & 97.8 & Sex & 0.046 & 1 & 0.83 \\
\hline IV & 43 & 2 & 95.6 & Instar moved & 73.9 & 4 & $<0.001$ \\
\hline $\mathrm{V}$ & 22 & 34 & 39.3 & & & & \\
\hline \multicolumn{8}{|l|}{ A. levana } \\
\hline \multicolumn{8}{|c|}{ Long day $\rightarrow$ short day } \\
\hline I & 0 & 29 & 0 & & & & \\
\hline II & 0 & 29 & 0 & Sex & 0.68 & 1 & 0.41 \\
\hline III & 0 & 28 & 0 & Instar moved & 126.5 & 4 & $<0.001$ \\
\hline IV & 11 & 18 & 37.9 & & & & \\
\hline $\mathrm{V}$ & 27 & 0 & 100 & & & & \\
\hline \multicolumn{8}{|c|}{ Short day $\rightarrow$ long day } \\
\hline I & 30 & 0 & 100 & & & & \\
\hline II & 26 & 0 & 100 & Sex & 0.14 & 1 & 0.71 \\
\hline III & 29 & 0 & 100 & Instar moved & 54.8 & 4 & $<0.001$ \\
\hline IV & 29 & 0 & 100 & & & & \\
\hline $\mathrm{V}$ & 15 & 15 & 50 & & & & \\
\hline \multicolumn{8}{|c|}{ P. aegeria } \\
\hline \multicolumn{8}{|c|}{ Long day $\rightarrow$ short day } \\
\hline II & 0 & 10 & 0 & $\operatorname{Sex}^{\mathrm{a}}$ & 7.45 & 1 & 0.0063 \\
\hline III & 0 & 22 & 0 & Instar moved & 57.1 & 2 & $<0.001$ \\
\hline IV & $7^{\mathrm{a}}$ & $24^{\mathrm{a}}$ & 22.6 & & & & \\
\hline \multicolumn{8}{|c|}{ Short day $\rightarrow$ long day } \\
\hline II & 12 & 0 & 100 & Sex & $\mathrm{NA}^{\mathrm{b}}$ & NA & NA \\
\hline III & 28 & 0 & 100 & Instar moved & NA & NA & NA \\
\hline IV & 22 & 0 & 100 & & & & \\
\hline
\end{tabular}


Fig. 1 Comparison of the asymmetric ability to change pathway to fit the new environment between larvae transferred from a diapauseinducing condition (short daylength) into an environment that triggers direct development (long daylength) (filled squares) and larvae transferred in the opposite direction (open circles) in a $P$. napi, b A. levana and c $P$. aegeria. Data shown are the proportions of larvae ( \pm binomial $95 \% \mathrm{CI}$ ) that managed to adjust to the new environment when transferred between environments in the penultimate and ultimate larval instars

for P. napi and A. levana, sex did not affect the pathway decision of larvae that were transferred to the short daylength. Male $P$. aegeria entered diapause more easily than females when they reached the short daylength in the last instar (see Table 1 for statistics).

\section{P. napi}

Pieris napi larvae of both sexes (Table 1) behaved similarly in both replicates, and the pathway decision was strongly dependent on the timing of the transfer between cabinets (Table 1). Larvae that were transferred to their final cabinet as eggs or in instars I-III typically followed the larval pathway triggered in that final cabinet, as did the majority of larvae transferred in instar IV (Table 1). Larvae that were transferred in instar V typically did not switch their developmental response in accordance with the new daylength to which they had been transferred (Table 1). There was no difference in the ability to adjust to the new environment between the different sexes $\left(\chi_{1}^{2}=0.36\right.$, $P=0.85)$ or replicates $\left(\chi_{1}^{2}=0.81, P=0.37\right)$, and these factors were thus removed from the model. Instead, the larval potential to respond to the new daylength depended on the transfer direction, and larvae that came from a long daylength into a short daylength were typically more reluctant to respond to the new environment by entering diapause development. By contrast, most larvae that were moved from the short to the long daylength in the penultimate instar switched pathways and entered direct development, and a substantial fraction (39\%) were able to adjust their decision to the new daylength when the transfer took place as late as in the last instar (instar moved $\chi_{1}^{2}=95.3, \quad P<0.001 ; \quad$ transfer direction $\chi_{1}^{2}=22.4$, $P<0.001 ;$ all interactions were nonsignificant and removed from the model; Fig. 1a).

\section{A. levana}

The pattern exhibited by A. levana was almost identical to that of $P$. napi; invariably, all larvae made their pathway choice according to the novel environment when transferred there in instars I-III. The majority of larvae transferred in the fourth instar also had the ability to adjust their decision to the new conditions, whereas the majority of
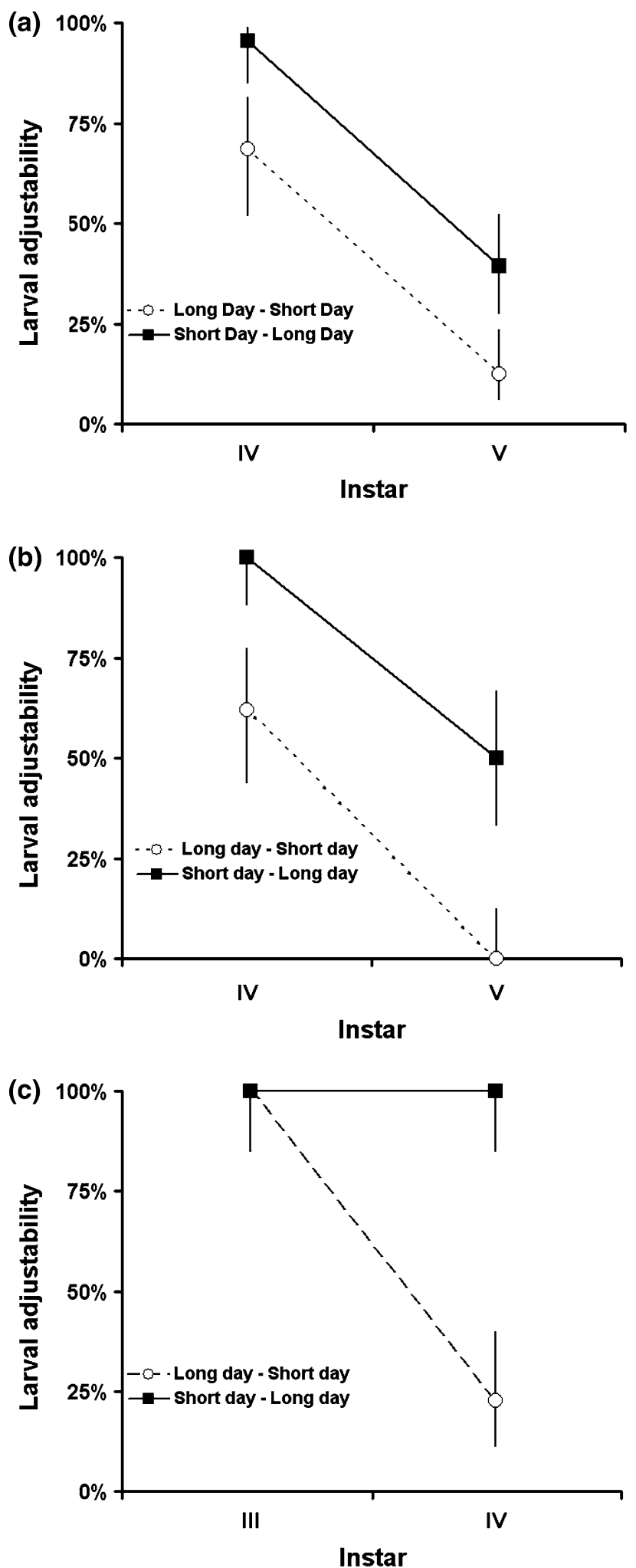

larvae transferred in instar $\mathrm{V}$ did not alter their decision to fit the new environment (Table 1). Furthermore, as in P. napi, A. levana larvae more easily shifted their decision in order to enter direct development than vice versa, as 
about half of the larvae transferred from the short day to the long day treatment adjusted to the new environment as late as in the fifth instar and entered diapause, whereas none of the larvae that were transferred from long day to short day conditions shifted their continued development in order to enter diapause (instar moved $\chi_{1}^{2}=69.5, P<0.001$; transfer direction $\chi_{1}^{2}=42.9, P<0.001$; sex $\chi_{1}^{2}=0.40, P=0.40$, and all initial interactions involving sex were nonsignificant and removed from the model; Fig. 1b).

\section{P. aegeria}

The decision-making pattern in $P$. aegeria differed from those of $P$. napi and A. levana in that the larval pathway decision was made in the ultimate (IV) instar. Individuals transferred between the long day and the short day treatment in the third (penultimate) instar invariably made the appropriate pathway decision for the novel conditions. In fact, all larvae transferred from short day to long day conditions in the ultimate instar switched pathways to undergo direct development (Table 1). However, a substantial fraction of the larvae transferred in the opposite direction were unable to adjust to the new environment and entered direct development despite the short daylength in the new climate cabinet. Hence, the larval potential to adjust to the new environment was affected by both how late in life the larvae were transferred (instar moved $\left.\chi_{1}^{2}=45.9, P<0.001\right)$ and the direction in which they were transferred (short day to long day or vice versa; transfer direction $\left.\chi_{1}^{2}=41.5, P<0.001\right)$. Furthermore, a smaller fraction of the females than males entered diapause when transferred to the short daylength in the ultimate instar ( $\left.\operatorname{sex} \chi_{1}^{2}=7.5, P=0.0063\right)$. Replicate $\left(\chi_{1}^{2}=0.128\right.$, $P=0.72)$, population (southern/northern) $\quad\left(\chi_{1}^{2}=3.25\right.$, $P=0.07)$ and all interactions were nonsignificant and removed from the model.

\section{Discussion}

$P$. napi and A. levana larvae typically make the decision whether to enter direct or diapause development in one of the last two larval instars (IV, V), whereas $P$. aegeria decides whether to enter diapause or to continue development in the ultimate (IV) instar. The ability to switch developmental pathways when the environmental cues change is, however, conditional; in all three species, larvae can switch from diapause to direct development later in their development than they can make the reverse switch. This strongly implies a general difficulty in making the decision to enter diapause development late in the larval period, which is probably explained by the extra preparations needed to survive a long and cold winter compared with those needed in order to start reproductive life within 1-2 weeks after pupation.

There are several possibilities that can explain the greater ability of $P$. aegeria larvae to adjust their pathway decisions well into the ultimate instar. The two other species studied here, $P$. napi and A. levana, both have five larval instars, whereas $P$. aegeria develops through four larval stages. Hence, larvae of this species spend relatively more time in their ultimate instar, and they might therefore enter their ultimate instar proportionally earlier in their development. Furthermore, as described above (see "Materials and methods"), this species can also diapause as larvae. A recent study (Wiklund and Friberg 2010) shows that larval diapause is possible only in the third instar; whereas most larvae survive the winter when incubating at a low temperature in the third instar, no larvae survived when the cold period hit them in the fourth instar. Therefore, before the end of the third instar, larvae of this species have to make the choice of whether to enter larval diapause or continue development to the pupal stage, and this in turn means that the decision whether to continue development into the reproductive stage or enter pupal diapause must wait until the ultimate instar, when larval diapause is no longer an option. Interestingly, males appeared to reverse a decision to develop directly instead of entering diapause development more easily than females when they where transferred to the short day treatment in the ultimate instar, and future studies are needed to determine whether this is an artefact of the limited sample size or whether it reflects sex-specific life-history demands acting on this species.

A. levana shows little individual variation within each treatment. The pathway decision varied only in the cohorts of larvae transferred from the long day to the short day in the fourth instar and larvae transferred in the opposite direction in the fifth instar. This implies that it is possible to shift from the direct development pathway to diapause in the fourth instar, and from a diapause pathway to direct development as newly moulted fifth instar larvae, and that there is little genetic variation in the timing of the pathway decision in this species as well. As in P. napi, the decision whether to enter diapause or direct development is made in one of the last two larval instars, but the data show more variation in the timing of the decision; while a few larvae that were transferred between light regimes in the third instar larvae, weighing only 3-10 mg, had already made their pathway decisions, other larvae could adjust their decisions in both transfer directions as late as a few days into the fifth instar, when they weighed just a little less than $100 \mathrm{mg}$. The larger variation in the timing of the decision point in this species could arguably imply a release of selection pressure on the pathway decision in this species in the Stockholm area. However, during some years 
(Eliasson et al. 2005), this species appears in a partial third generation in late August and September, and the risk of making the wrong decision by continuing direct development too late in the season is likely to select on the decision-making procedure in this area. It is therefore more likely that the somewhat larger variation in decision time present in $P$. napi might reflect a pattern of dispersal, with genes immigrating from other populations that are locally adapted to latitudes southern (or northern) to the one in south-central Sweden, where a $16 \mathrm{~h}$ daylength might indicate a time of year when it is still favourable to develop directly without diapause.

During winter, the diapausing insects need to survive both cold temperatures and high levels of dehydration (e.g. see Danilevskii 1965; Hodkova and Hodek 2004 for reviews) as well as up to 10 months of inactivity with no chance of escaping potential predators. In order to do this they need to be internally prepared by physiological alterations (e.g. see reviews in Danilevskii 1965; Hodkova and Hodek 2004; Koštál 2006), and an overwintering pupa typically has, for example, a thicker cuticle (Yata et al. 1984) and denser tissue (Kaneko and Katagiri 2006) than a directly developing one. We contend that the difficulties of switching from being set for direct development into entering diapause act generally on all pupal diapausers, and thus can be predicted to be a general pattern in butterflies as well as in other insects. The laboratory conditions experienced by the larvae in this study are of course artificial, and in nature no larvae would experience such dramatic decreases or increases in daylength during development. Still, this study confirms that the decision is not made at a well-defined point in time as much as during a short period of susceptibility, as shown by the asymmetry of the larval pathway decision and the ability of larvae set for diapause to alter their decisions-sometimes as late as well into the fifth instar. Hence, the decision points in the penultimate or ultimate instar (depending on the transfer direction and species) detected in this study should be seen as points of no return rather than times when the decision is made.

The similar timing of the point of no return among the three species implies that natural selection favours a decision made late in larval development, when larvae have up-to-date information about their position in the season. The decision asymmetry-it appears to be more difficult to revert a conditional decision to develop directly instead of entering diapause than vice versa-is, however, most probably better described as a developmental constraint. The decision to diapause must be made earlier in development so that the growing larva has time to make the appropriate physiological preparations needed to survive the many months until the following spring. After a certain point in time, diapause is likely no longer an option if these preparations have not been initiated. The possible presence of conditional decisions made before the final decision point calls for further studies. For example, such studies could focus on the extent to which larvae make growth adjustments in accordance with these decisions (increase developmental rate if they choose to continue development/decrease developmental rate if they decide to diapause), or whether the faster growth rate of direct developers under critical conditions is actually an intrinsic cue, so that only those larvae that grow the fastest have the ability to enter direct development when time stressed.

The pattern detected in this study-that the final decision between diapause and direct development is made in the penultimate or ultimate instar-also has implications for studies on decision-making in relation to seasonal polyphenism. All of these species have been used as model organisms for seasonal polyphenism (e.g. Fric and Konvicka 2002; Van Dyck and Wiklund 2002; Karlsson and Johansson 2008), and A. levana is certainly one of the most extreme examples of seasonal polyphenism in nature. Apart from their striking differences in colouration, the two generations also differ in body design, wing loading, dispersal propensity and resource allocation pattern (Fric and Konvicka 2002; Friberg and Karlsson 2010). The spring and summer generations of $P$. napi differ in dispersal ability and fecundity (Karlsson and Johansson 2008), as well as in mating propensity and sexual maturity at eclosion (Larsdotter Mellström et al. 2010), whereas the different developmental pathways of $P$. aegeria differ in the number of eyespots present on the dorsal side of the hind wing (Shreeve 1987), as well as in body size and fecundity (Van Dyck and Wiklund 2002; Gotthard and Berger 2010). All these adaptive or nonadaptive, season-specific traits must be developed downstream of the decision whether to enter diapause or direct development, which may further explain why there is a point of no return after which the larvae cannot switch pathways after a certain size or age. We contend that the findings in this study may be important for future studies investigating the mechanisms of seasonal polyphenism, which at this point have not unravelled the extent to which the induction of diapause or direct development functions as a developmental switch that influences a suite of traits (see above), or whether the induction mechanism for the generation-specific polyphenic traits is disentangled from the pathway decision-making mechanism and induced separately, although typically coinciding with the pathway decision in nature. 
Acknowledgments We thank Bengt Karlsson, David Berger, Wolf Blanckenhorn, Toomas Tammaru and one anonymous reviewer for input on earlier drafts of this manuscript. This study was financed by grants from the Swedish Research Council to K.G. and C.W.

Open Access This article is distributed under the terms of the Creative Commons Attribution Noncommercial License which permits any noncommercial use, distribution, and reproduction in any medium, provided the original author(s) and source are credited.

\section{References}

Abrams PA, Leimar O, Nylin S, Wiklund C (1996) The effect of flexible growth rates on optimal sizes and development times in a seasonal environment. Am Nat 147:381-395

Alcock J (2009) Animal behavior: an evolutionary approach, 9th edn. Sinauer Associates Inc., Sunderland

Andersson M (1994) Sexual selection (Monographs in Behavior and Ecology). Princeton University Press, Princeton

Barker RJ, Mayer A, Cohen CF (1963) Photoperiod effects in Pieris rapae (L.). Ann Entomol Soc Am 56:292-294

Carriere Y, Simons AM, Roff DA (1996) The effect of the timing of post-diapause egg development on survival, growth, and body size in Gryllus pennsylvanicus. Oikos 75:463-470

Danilevskii AS (1965) Photoperiodism and seasonal development of insects. Oliver and Boyd, London

Danks HV (1987) Insect dormancy: an ecological perspective. Biological Survey of Canada (Terrestrial Arthropods), Ottawa

Danks HV (2007) The elements of seasonal adaptations in insects. Can Entomol 139:1-44

Eizaguirre M, Lopez C, Asín L, Albajes R (1994) Thermoperiodism, photoperiodism, and sensitive stage in the diapause induction of Sesama nonagrioides (Lepidoptera: Noctuidae). J Insect Physiol 40:113-119

Eliasson CU, Ryrholm N, Holmer M, Jilg K, Gärdenfors U (2005) Nationalnyckeln till Sveriges flora och fauna Fjärilar: Dagfjärilar Hesperiidae-Nymphalidae. Artdatabanken SLU, Uppsala (in Swedish)

Friberg M, Karlsson B (2010) Life history polyphenism in the map butterfly (Araschnia levana) - developmental constraints vs season-specific adaptations. Evol Ecol Res (in press)

Friberg M, Wiklund C (2009) Host plant preference and performance of the sibling species of butterflies Leptidea sinapis and Leptidea reali: a test of the trade-off hypothesis for food specialization. Oecologia 159:127-137

Friberg M, Wiklund C (2010) Host plant induced larval decisionmaking in a habitat/host plant generalist butterfly. Ecology 91:15-21

Friberg M, Bergman M, Kullberg J, Wahlberg N, Wiklund C (2008) Niche separation in space and time between two sympatric sister species-a case of ecological pleiotropy. Evol Ecol 22:1-18

Fric Z, Konvicka M (2002) Generations of the polyphenic butterfly Araschnia levana differ in body design. Evol Ecol Res 4:1017-1032

Gotthard K (1998) Life history plasticity in the satyrine butterfly Lasiommata petropolitana: investigating an adaptive reaction norm. J Evol Biol 11:21-39

Gotthard K (2008) Adaptive growth decisions in butterflies. Bioscience 8:222-230

Gotthard K, Berger D (2010) The diapause decision as a cascade switch for adaptive developmental plasticity in body mass in a butterfly. J Evol Biol 23:1129-1137
Hodkova M, Hodek I (2004) Photoperiod, diapause and coldhardiness. Eur J Entomol 101:445-458

Hunter MD, McNeil JN (1997) Host-plant quality influences diapause and voltinism in a polyphagous insect herbivore. Ecology 78:977-986

Jackson WH (1890) Studies in the morphology of the Lepidoptera. Part I. Linn Soc London Transl Ser 2 Zool 5:143-186

Jaenike J (1978) On optimal oviposition behavior in phytophagous insects. Theor Popul Biol 14:350-356

Kaneko J, Katagiri C (2006) A simple method to discriminate diapause from non diapause pupae in large and small white butterflies, Pieris brassicae and P. rapae crucivora. Naturwissenschaften 93:393-396

Karlsson B, Johansson A (2008) Seasonal polyphenism and developmental trade-offs between flight ability and egg laying in a pierid butterfly. Proc R Soc B 275:2131-2136

Kingsolver JG (1995) Fitness consequences of seasonal polyphenism in western white butterflies. Evolution 49:942-954

Koevos DS, Tzanakakis ME (1991) Effect of hydration, photoperiod and temperature on diapause termination in eggs of Petrobia (Tetranychina) harti (Acari: Tetranychidae). Exp Appl Acarol 11:111-123

Koštál V (2006) Eco-physiological phases of insect-diapause. J Insect Physiol 52:113-127

Krebs JR, Davies NB (1993) An introduction to animal behaviour, 3rd edn. Oxford University Press, Oxford

Larsdotter Mellström H, Friberg M, Borg-Karlson A-K, Murtazina R, Palm M, Wiklund C (2010) Seasonal polyphenism in life history traits: time costs of direct development in a butterfly. Behav Ecol Sociobiol 64:1377-1383

McNamara JM, Houston AI (1996) State-dependent life histories. Nature 380:215-221

Mikkola K (2004) The red admiral butterfly (Vanessa atalanta, Lepidoptera: Nymphalidae) is a true seasonal migrant: an evolutionary puzzle resolved? Eur J Entomol 100:625-626

Moran NA (1992) The evolution of life cycles in aphids. Ann Rev Entomol 37:321-348

Nesbit RL, Hill JK, Woiwod LP, Sivell D, Bensusan KJ, Chapman JW (2009) Seasonally adaptive migratory headings mediated by a sun compass in the painted lady butterfly, Vanessa cardui. Anim Behav 78:1119-1125

Nordström F (1955) Distribution of Fennoscandian butterflies. K Fysiogr Sällsk Handl (N.F.) 66:1-175 (in Swedish)

Nylin S (1992) Seasonal plasticity in life history traits: growth and development in Polygonia c-album (Lepidoptera: Nymphalidae). Biol J Linn Soc 47:301-323

Saulich AK, Musolin DL (1996) Univoltinism and its regulation in some temperate true bugs (Heteroptera). Eur $\mathrm{J}$ Entomol 93:507-518

Shapiro AM (1976) Seasonal polyphenism. Evol Biol 9:259-333

Shintani Y, Higuchi H (2008) Developmental parameters and photoperiodism in Trigonotylus tenuis (Reuter) (Heteroptera: Miridae). Appl Entomol Zool 43:259-264

Shreeve TG (1987) The mate location behaviour of the male speckled wood butterfly, Pararge aegeria, and the effect of phenotypic differences in hind-wing spotting. Anim Behav 35:682-690

Solbreck C (1979) Induction of diapause in a migratory seed bug Neacoryphus bicrucis (Say) (Heteroptera: Lygaeidae). Oecologia 43:41-49

Stearns SC (1992) The evolution of life histories. Oxford University Press, Oxford

Stearns SC, Hoekstra RF (2000) Evolution: an introduction. Oxford University Press, Oxford

Stefanescu C (2001) The nature of migration in the red admiral butterfly Vanessa atalanta: evidence from the population ecology in its southern range. Ecol Entomol 26:525-536 
Stefanescu C (2007) Migration of the painted lady butterfly, Vanessa cardui, to north-eastern Spain is aided by African wind currents. J Anim Ecol 76:888-898

Stephens DW, Krebs JR (1986) Foraging theory (Monographs in Behavior and Ecology). Princeton University Press, Princeton

Tauber MJ, Tauber CA, Mazaki S (1986) Seasonal adaptations of insects. Oxford University Press, New York

Van Dyck H, Wiklund C (2002) Seasonal butterfly design: morphological plasticity among three developmental pathways relative to sex, flight and thermoregulation. J Evol Biol $15: 216-225$

Wedell N, Nylin S, Janz N (1997) Effects of larval host plant and sex on the propensity to enter diapause in the comma butterfly. Oikos 78:569-575

West-Eberhard MJ (2003) Developmental plasticity and evolution. Oxford University Press, New York

Wiklund C, Friberg M (2010) Seasonal development and variation in abundance among four annual flight periods in a butterfly-a 20 year study of the speckled wood (Pararge aegeria). Biol J Linn Soc (in press)

Wiklund C, Nylin S, Forsberg J (1991) Sex-related variation in growth-rate as a result of selection for large size and protandry in a bivoltine butterfly, Pieris napi. Oikos 60:241-250

Wiklund C, Wickman P-O, Nylin S (1992) A sex difference in the propensity to enter direct/diapause development: a result of selection for protandry. Evolution 46:519-528

Wiklund C, Vallin A, Friberg M, Jakobsson S (2007) Rodent predation on hibernating peacock and small tortoiseshell butterflies. Behav Ecol Sociobiol 62:379-389

Windig JJ, Lammar P (1999) Evolutionary genetics of seasonal polyphenism in the map butterfly Araschnia levana (Nymphalidae: Lepidoptera). Evol Ecol Res 1:875-894

Yata O, Saigusa T, Nakanishi A, Shima H (1984) Seasonal polyphenism in four Japanese Pieris (Artogeia) species. In: Vane-Wright RI, Ackery PR (eds) The biology of butterflies. Academic, London, pp 333-353 